\section{EDITORIAL}

TOMO 59

Número 6

Julio-Agosto

2006

\section{ARCHIVOS ESPAÑOLES DE UROLOGIA}

FUNDADOS POR E. PEREZ CASTRO, A. PUIGVERT GORRO Y

L. CIFUENTES DELATTE

Director / Editor: E. Pérez-Castro Ellendt

Editor Asociado: L. Martínez-Piñeiro Lorenzo

\title{
CANCER DE PRÓSTATA: SOBRETRATAMIENTO Y SOBREDIAGNÓSTICO
}

Ricardo García González y Teodoro Mayayo Dehesa²

Servicios de Anatomía Patológical y Urología². Hospital "Ramón y Cajal". Madrid. España.

RESUMEN: Desde la popularización del test sérico del PSA, la incidencia del diagnóstico del cáncer de próstata y su detección como enfermedad órgano-confinada han aumentado significativamente, disminuyendo, solo ligeramente, su tasa de mortalidad. La evidencia de que la mayoría de los varones con cáncer prostático diagnosticado en un screening de PSA mueren por diferente causa a su enfermedad ha condicionado los conceptos de sobretratamiento y sobrediagnóstico del mismo. El patólogo, que realiza el diagnóstico, tiene la responsabilidad de no sobrediagnosticar el cáncer, extremando su prudencia ante los focos tumorales minúsculos (en nuestra serie, el $8,57 \%$ de los tumores ocupaban menos del 3\% del total del tejido de la biopsia) y los cambios histológicos de valoración dudosa, especialmente los de ASAP (AGF), así como los patrones tumorales 1 y 2 de Gleason que, en nuestra opinión (tras el estudio de más de 9.000 casos), son de difícil o imposible valoración en los cilindros de biopsia. Y el urólogo debe hacer una interpretación clínica de esos mínimos tumores, o de las lesiones sospechosas, en conjunción con las condiciones clínicas de los pacientes antes de establecer una actitud terapéutica, evitando el sobretratamiento.

\section{A mediados de los años 80 se introdujo en la practica clínica la determinación séri-} ca del PSA como método de detección del cáncer de la próstata y se desarrolló la técnica de obtención de biopsias prostáticas en sextantantes mediante agujas finas dirigidas con ecografía transrectal, utilizando un procedimiento semiautomático (Bioptyß) para su realización. Estas dos circunstancias, unidas a la creciente demanda social de detección de la enfermedad cancerosa en el periodo en el que la neoplasia es mínimamente invasiva, han originado un notable aumento en el número de diagnósticos de carcinoma prostático y una modificación de las características clínicas y patológicas de la enfermedad en el momento del diagnóstico. 
Incidencia: En los EEUU, la incidencia anual del cáncer de próstata en los años 80 era de alrededor de 69 casos/ 100.000 varones; en el año 2000, fue de 104 casos $(1,2)$. Es decir, en esos 20 años la incidencia del diagnóstico del cáncer prostático casi se duplicó, lo que hace pensar que alrededor del $50 \%$ de los casos solo se diagnosticaron a partir del screening con PSA. Cifras similares se han comunicado en algunos estudios españoles $(3,4)$ y europeos $(5,6)$.

Morbilidad: Desde la introducción del test del PSA la mayoría de los cánceres prostáticos se detectan en periodo pre-clínico, asintomático. El porcentaje de tumores encontrados con enfermedad metastásica era, antes de la popularización del test, del $20 \%$, sin embargo en los últimos años este porcentaje se ha reducido al $5 \%$ (7).

Mortalidad: Se asume que la mayoría de los hombres con cáncer de próstata diagnosticados en un screening morirán por una causa ajena al mismo (8-10). En un estudio comparativo de dos poblaciones similares, pero con diferente grado de screening y tratamiento radical del cáncer de próstata, no se encontraron diferencias significativas en la tasa de mortalidad debida a este tumor (11). Pero en los EEUU, en la era pre-PSA, el cáncer de próstata era responsable del $9 \%$ de las muertes de los varones $y$, actualmente, es del 3\% (12), esto significa que la detección precoz de la enfermedad y su tratamiento, realmente reducen su tasa de mortalidad en, aproximadamente, un $6 \%$. No obstante, según el estudio de Nicholson y Harland (13), un paciente-tipo (65 años; Gleason 6) tendría un $11 \%$ de riesgo máximo de morir a causa de su cáncer prostático en 15 años, y un $44 \%$ por otras causas diferentes. Aplicando los criterios del Scandinavian Prostatic Cancer Group (14), el tratamiento radical del mismo paciente-tipo disminuiría el riego máximo de muerte por el tumor únicamente al 7\%, es decir, una reducción del $4 \%$.

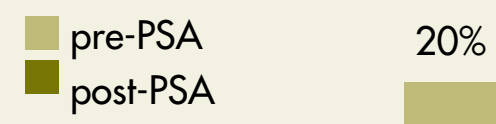

104

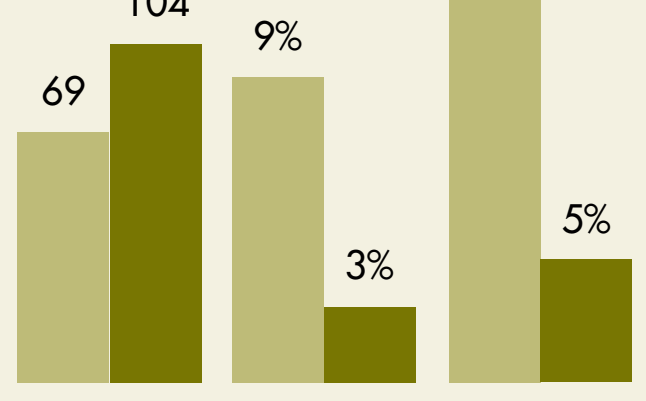

Tratamiento: Mientras que a los pacientes con otro tipo de cáncer curable se les ofrece, inmediatamente al diagnóstico, un tratamiento radical, en el manejo del cáncer prostático localizado, hasta los años 80 , se consideraba que un "seguimiento expectante" era una actitud alternativa con resultados aceptables para algunos pacientes. Sin embargo, en un estudio a 10 años, el riesgo de enfermedad metastásica fue del $13 \%$ en los paciente prostatectomizados, frente al $27 \%$ en los que se siguió una pauta de "seguimiento expectante" (14); en consecuencia ya no es defendible esta actitud. Podría aceptarse el tratar todos los casos, incluso aquellos que nunca ocasionarían síntomas, si el tratamiento no tuviera secuelas; pero el tratamiento del cáncer de próstata, en todas sus modalidades, tiene complicaciones que pueden afectar seriamente la calidad de vida 
del paciente sin aumento de su supervivencia (15), por lo que la terapia debería estar restringida a aquellos pacientes que realmente la necesitaran.

A pesar de todo y puesto que, actualmente, no podemos predecir la evolución de un cáncer dado, la forma convencional de enfrentarnos a la enfermedad detectada en un screening es, generalmente, la de ofrecer un tratamiento curativo a todos los varones con enfermedad localizada (16) aún sabiendo que, en muchos casos, este tratamiento no es necesario. Esta actitud no es la ideal, de forma que el establecimiento del tratamiento radical se hace más difícil de mantener a medida que se generaliza el uso del test del PSA y el número de casos de cáncer aumenta, sobre todo en aquellos pacientes que, de no haber sido seleccionados mediante la determinación del PSA sérico y biopsiados posteriormente, nunca hubieran manifestado clínicamente la enfermedad.

Sobretratamiento: Teniendo presente que el cáncer de próstata tiene su mayor impacto en el subgrupo de población con menor expectativa de vida -el $90 \%$ aparecen en varones mayores de 65 años (17)- y puesto que la mayoría de los pacientes tienen buen pronóstico, algunos autores han planteado que estos tumores se están tratando innecesariamente, y sostienen un concepto emergente, el de "sobretratamiento" del cáncer de próstata $(18,19)$.

Lo importante para el manejo del cáncer prostático sería, por un lado, identificar aquellos enfermos que requieren el tratamiento en un momento en el que todavía son curables y se van a beneficiar del mismo; por otro, evitar tratar a los que tienen la enfermedad indolente, destinada a ser un mero hallazgo histológico incidental; $y$, finalmente, seleccionar los que son incurables, en los que la terapia radical será ineficaz. Es necesario establecer un equilibrio entre la detección precoz del tumor y la aplicación selectiva de la terapia. La utilización de los marcadores, en suero y en tejido, y de las nuevas técnicas de imagen, hace que los pacientes puedan agruparse mejor según el riesgo de progresión y metástasis (20), pero aún se necesita encontrar mejores métodos de selección de aquellos enfermos que pueden beneficiarse con un tratamiento radical.

Sobrediagnóstico: En los últimos años se ha planteado el problema del sobrediagnóstico del cáncer de próstata, entendido como "la detección del cáncer de próstata mediante el test de PSA que de otra forma no se hubiera diagnosticado en la vida del paciente" $(8,9,21)$. El concepto está basado en la evidencia, ya mencionada, de que muchos de los tumores detectados mediante campañas de screening con la determinación de PSA sérico y su posterior diagnóstico mediante biopsias dirigidas con ecografía transrectal, no se hacen sintomáticos durante la restante vida del enfermo, aún sin tratamiento, y mueren por una causa diferente a su cáncer prostático $(8,9,10)$.

Patología: Puesto que el patólogo es el especialista que decide el diagnóstico del tumor, asume el protagonismo en el problema del sobrediagnóstico y participa en el de sobretratamiento. El diagnóstico del cáncer clínicamente insignificante en una biopsia prostática con aguja parece que no es infrecuente y puede tener consecuencias desafortunadas para los pacientes ya que suele conducir a un tratamiento con morbilidad asociada que puede no ser necesario (18). Además del diagnóstico del tumor, es necesario valorar aquellos datos patológicos que puedan influir en la toma de decisiones terapéuticas. Ya que solo aquellos tumores que poseen líneas celulares con alta tasa de crecimiento y agresividad obtendrían beneficio de un diagnóstico y tratamiento precoz 
(22), sería de gran valor, en la práctica diaria, tener un marcador objetivo, sensible y específico para la identificación del tumor agresivo y en este sentido se orientan las recientes investigaciones, $(23,24)$.

Muchos de los factores predictivos en el cáncer de próstata son derivados del estudio microscópico del tumor. Dos Conferencias Internacionales de Consenso, celebradas en 1999 y patrocinadas por Colegio de Patólogos Americanos (CAP) y la OMS, han establecido los factores pronósticos para el uso rutinario en las piezas de prostatectomía radical, $(25,26)$. Sin embargo, no hay factores suficientemente desarrollados para su aplicación en las biopsias por aguja. Generalmente se consideran como válidos la puntuación de Gleason y la estimación del tamaño del tumor, basada en la cantidad de masa tumoral existente en las muestras (número de cilindros afectados y proporción de tejido neoplásico sobre el total biopsiado, o la dimensión de los focos tumorales en relación con la de los cilindros) $(27,28)$.

El sistema de Gleason, que ha supuesto una forma universal de unificación de los datos histológicos del adenocarcinoma prostático, se ha mostrado como un método en el que, aunque tenga un aceptable grado de reproductibilidad (29), la subjetividad y el entrenamiento específico de los patólogos influyen en su valoración (15). Basados en nuestra experiencia del estudio de más de 9.000 biopsias en ecodirigidas creemos, con otros autores (30), que en este tipo de biopsias un adenocarcinoma con una valoración de Gleason menor de 5 lo más probable es que esté infragradado o que se trate de una lesión benigna simuladora de cáncer.

Actualmente, las características patológicas de la enfermedad han cambiado substancialmente. La drástica disminución de cánceres de próstata diagnosticados como enfermedad sintomática y generalmente metastásica, se ha visto sustituida por una mayor proporción de tumores órgano-confinados, con una reducción significativa de la carga tumoral en la glándula, a menudo, con una distribución microscópica multifocal. Está establecido que el tamaño del tumor encontrado en las biopsias con aguja no siempre refleja el que aparece en la pieza de prostatectomía, aunque sí lo hace en un notable porcentaje de los casos (31). Hoy en día se encuentra un importante aumento de biopsias en las que hay un pequeño foco de adenocarcinoma. Su identificación es unos de los mayores retos diagnósticos en la patología quirúrgica, aun con la utilización complementaria de los marcadores inmunohistoquímicos de células basales (CK903 y p63) y de células neoplásicas (P504S) (32,33). En una revisión de nuestra casuística, de entre 4.064 biopsias prostáticas ecodirigidas consecutivas encontramos $1.493(36,74 \%)$ carcinomas, de los cuales 128 $(8,57 \%)$ tienen una afectación unilateral, focal y menor del $3 \%$ del total del tejido prostático biopsiado. Aproximadamente el $20 \%$ de las biopsias con aguja tienen menos del $5 \%$ del tejido de los cilindros de biopsia afectado por la neoplasia.

Aquellos tumores, con puntuación de Gleason menor de 7 y poca masa tumoral en la biopsia, tanto por su probable pequeño tamaño como por su buena diferenciación, son neoplasias sin probable significado clínico en la restante vida del paciente. Pero hay casos excepcionales, en los que un tumor, con todos los criterios favorables en la biopsia, se ha mostrado con afectación masiva en la pieza de prostatectomía, incluso, con extensión extraprostática. Esta situación podría ser debida a la localización primaria del tumor en la parte anterior de zona de transición o de 
la periférica, con crecimiento hacia la lámina anterior prostática; estas localizaciones no suelen ser objetivo de la biopsia, por lo que el tumor puede no estar suficientemente representado en los cilindros obtenidos. Esta circunstancia se acrecienta en aquellos pacientes en los que coexiste una HBP de volumen suficiente.

El diagnóstico diferencial de los pequeños focos tumorales incluye un número de lesiones glandulares benignas (hiperplasia postatrófica, adenosis esclerosante, etc. ) que pueden confundirse con un carcinoma. En ocasiones, su diagnóstico diferencial es difícil o imposible, habiéndose acuñado la denominación de "Atipical Small Acinar Proliferation (ASAP)" (34) para describir focos microacinares sospechosos pero no conclusivos de malignidad. A estos cambios microscópicos, que nosotros denominamos "Atipia Glandular Focal (AGF)" (35), puesto que no siempre la lesión es solo microacinar ni siempre se puede reconocer como una lesión proliferativa, y que en nuestra serie suponen un $2,41 \%$ (98 casos) del total de las biopsias, se les atribuye un alto valor predictivo de cáncer $(35,36)$, (superior al del PIN-AG). Esta situación no se ha resuelto con la ayuda de los métodos inmunohistoquímicos, si no es para segregar aquellas lesiones que presenten inmunoreactividad de células basales, que las definen como de naturaleza benigna.

Nuestro criterio es que cuando se encuentra un foco diminuto de evidente adenocarcinoma, en la mayoría de los casos, la lesión tiene un patrón 4 o 5 de Gleason con lo que el tumor tendrá, al menos, una puntuación 7 , es decir será un tumor considerado como potencialmente agresivo. Pero los mínimos focos de patrón 3 de Gleason (puntuación 6) y, desde luego, de patrón 2 (en cilindros de biopsia, consideramos muy difícil valorar los patrones 1 y 2 , si no imposible), deben ser valorados con extrema prudencia, ya que el diagnóstico puede condicionar un tratamiento radical de conocida morbilidad en un $50 \%$ de los casos y un beneficio de supervivencia de, únicamente, alrededor del $2 \%$. Siendo preferible, en ocasiones límites, su valoración como AGF, con repetición de la biopsia, al sobrediagnóstico de la lesión.

Con el diagnóstico de AGF, el paciente será sometido a nuevas biopsias que pueden o no demostrar un tumor evidente. Si se demuestra, el tumor muy probablemente tenga un tamaño o un grado de diferenciación suficiente para ser considerado un cáncer que necesita ser tratado. Si no, en el supuesto de la existencia oculta del tumor, posiblemente es lo suficientemente pequeño y lo suficientemente bien diferenciado como para no beneficiarse de un tratamiento radical. Estas consideraciones se hacen aún más válidas si la biopsia es repetida una o más veces (sabemos que, en ocasiones, tras el diagnóstico de AGF, el tumor no ha aparecido hasta la 5 a rebiopsia pero creemos que estos tumores son, generalmente, irrelevantes para vida del paciente). Los datos adicionales de PSA, DPSA y relación PSA libre/ PSA total, ayudan a la consideración de un cáncer prostático como poco significativo (37). Así que, el diagnóstico de tumores con mínima afectación y/o suma de Gleason menor de 6, o de las lesiones sospechosas de cáncer, debe ser realizado, en las BPES, con precaución, y su valoración clínica debe efectuarse en el contexto general del paciente antes de establecer actuaciones agresivas, con rebiopsias o terapia radical, que pueden no ser necesarias. 


\section{BIBLIOGRAFÍA y LECTURAS RECOMENDADAS}

1. YOUNG, J.L. (Jr); PERCY, C.L.; ASIRE, A.J. y cols.: "Cancer incidence and mortality in the United States, 1973-77". Natl. Cancer Inst. Monogr.; 57: 1. 1981.

2. McDAVID, K.; LEE, J.; FULTON, J.P. y cols.: "Prostate cancer incidence and mortality rates and trends in the United States and Canada". Public Health Rep; 119: 174. 2004.

3. HERRANZ AMO, F.; ARIAS FUNEZ, F.; ARRIZABALGA MORENO, M. y cols.: "El cáncer de próstata en la Comunidad de Madrid en el año 2000". I.-Incidencia. Actas Urol. Esp.; 27: 323. 2003.

4. BERENGUER, A.; LUJAN, M.; PAEZ, A. y cols.: "The Spanish contribution to the European Randomized Study of Screening for Prostate Cancer". BJU Int; 92 Suppl 2: 33. 2003.

5. CANTO, E.I.; SLAWIN, K.M.: "Early management of prostate cancer: how to respond to an elevated PSA?". Ann. Rev. Med.; 53: 355. 2002.

6. PARKER, C.: "Active surveillance: towards a new paradigm in the management of early prostate cancer". Lancet Oncol; 5: 101. 2004.

7. FARKAS, A.; SCHENEIDER, D.; PERROTTI, M. y cols.: "National trends in epidemiology of prostate cancer, 1973 to 1999: evidence for effectiveness of prostate-specific antigen screening". Urology; 52: 444. 1998.

8. YAO, S.L.; LU-YAO, G.: "Understanding and appreciating overdiagnosis in the PSA era". J. Natl. Cancer Inst.; 94: 958. 2002.

9. ETZIONI, R.; PENSON, D.F.; LEGLER, J.M. y cols.: "Overdiagnosis due to prostate-specific antigen screening: lessons from U.S. prostate cancer incidence trends". J. Natl. Cancer Inst.; 94: 981. 2002

*10. BRENNER, H.; ARNDT, V.: "Long-term survival rates of patients with prostate cancer in the prostate-specific antigen screening era: population-based estimates for the year 2000 by period analysis". J. Clin. Oncol.; 23: 441. 2005.

11. LU-YAO, G.; ALBERTSEN, P.C.; STANFORD, J.L. y cols.: "Natural experiment examining impact of aggressive screening and treatment on prostate cancer mortality in two fixed cohorts from Seattle area and Connecticut". BMJ; 325: 740. 2002.
12. CLEGG, L.X.; LI, F.P; HANKEY, B.F. y cols.: "Cancer survival among US whites and minorities: a SEER (Surveillance, Epidemiology, and End Results) Program population-based study". Arch Intern Med.; 162: 1985. 2002.

13. NICHOLSON, P.W.; HARLAND, S.J.: "Survival prospects after screen-detection of prostate cancer". BJU Int.; 90: 686. 2002.

14. HOLMBERG, L.; BILL-AXELSON, A.; HELGESEN, F. y cols.: "Scandinavian Prostatic Cancer Group Study Number 4. A randomized trial comparing radical prostatectomy with watchful waiting in early prostate cancer". N. Engl. J. Med.; 347: 781. 2002.

15. CHISM, D.B.; HALTON, A.L.; TRONCOSO, P. y cols.: "The Gleason score shift: score four and seven tears ago". Int. J. Radiation Oncology Biol. Phys.; 56: 1241. 2003.

16. FOWLER, F.J. (Jr); McNAUGHTON, COLLINS, M. y cols.: "Comparison of recommendations by urologists and radiation oncologists for treatment of clinically localized prostate cancer". JAMA; 283: 3217. 2000.

17. MEIKLE, A.W.; SMITH, J.A.: "Epidemiology of prostate cancer". Urol. Clin. North. Am.; 17: 709. 1990.

18. HAMMERER, P.: "pT0 after radical prostatectomy: Overtreatment for insignificant prostate cancer?". Eur. Urol.; 45: 35. 2004.

*19. CARROLL, P.R.: "Early stage prostate cancer-Do we have a problem with over-detection, overtreatment or both?". J. Urol.; 173: 1061. 2005.

20. BAGNALL, S.; KLOTZ, L.: "Conservative versus radical therapy of prostate cancer: how have recent advances in molecular markers and imaging enhanced our ability to prognosticate risk?". Semin. Oncol.; 30: 587. 2003.

21. CIATTO, S.; GERVASI, G.; BONARDI, R. y cols.: "Determinig overdiagnosis by screening with DRE/TRUS or PSA (Florence pilot studies, 1991-1994)". Eur. J. Cancer; 41: 411. 2005

22. LIN, D.W.; LANGE, P.H.: "The epidemiology and natural history of prostate cancer. En: Lepor H, ed. Prostatic diseases". WB Saunders Co. Philadelphia.. 345. 2000.

23. CHIN, J.L.; REITER, R.E.: "Molecular markers and prostate cancer prognosis". Clin. Prostate Cancer; 3: 157. 2004.

24. MOUL, J.W.; MERSEBURGER, A.S.; SRIVASTAVA, S.: "Molecular markers in prostate cancer: the role in preoperative staging". Clin. Prostate Cancer; 1: 42. 2002. 
*25. BOSTWICK, D.G.; FOSTER, C.S.: "Predictive factors in prostate cancer: current concepts from the 1999 College of American Pathologists Conference on Solid Tumor Prognostic Factors and the 1999 World Health Organization Second International Consultation on Prostate Cancer". Semin Urol. Oncol.; 17: 222. 1999.

26. VERHAGEN, P.C.; TILANUS, M.G.; DE WEGER, R.A. y cols.: "Prognostic factors in localised prostate cancer with emphasis on the application of molecular techniques". Eur. Urol.; 41: 363. 2002.

27. QUINN, D.I.; HENSHALL, S.M.; BRENNER, P.C. y cols.: "Prognostic significance of preoperative factors in localized prostate carcinoma treated with radical prostatectomy: importance of percentage of biopsies that contain tumor and the presence of biopsy perineural invasion". Cancer; 97: 1884. 2003.

28. SAN FRANCISCO, I.F.; REGAN, M.M.; OLUMI, A.F. y cols.: "Percent of cores positive for cancer is a better preoperative predictor of cancer recurrence after radical prostatectomy than prostate specific antigen". J. Urol.; 171: 1492. 2004

29. ALLSBROOK, W.C. (Jr); MANGOLD, K.A.; JOHNSON, M.H. y cols.: "Interobserver reproducibility of Gleason grading of prostatic carcinoma: general pathologist". Hum. Pathol.; 32: 81. 2001.

*30. EPSTEIN, J.I.: "Gleason score 2-4 adenocarcinoma of the prostate on needle biopsy". Am. J. Surg. Pathol.; 24: 477. 2000

31. SEBO, T.J.; BOCK, B.J.; CHEVILLE, J.C. y cols.: "The percent of cores positive for cancer in prostate needle biopsy specimens is strongly predictive of tumor stage and volume at radical prostatectomy". J. Urol.; 163: 174. 2000.

32. EPSTEIN, J.I.: "Diagnosis and reporting of limited adenocarcinoma of the prostate on needle biopsy". Mod. Pathol.; 17: 307. 2004.

33. SHAH, R.B.; KUNJU, L.P.; SHEN, R. y cols.: "Usefulness of basal cell cocktail (34betaE12 + p63) in the diagnosis of atypical prostate glandular proliferations". Am. J. Clin. Pathol.; 122: 517. 2004.

34. ICZKOWSKI, K.A.; MACLENNAN, G.T.; BOSTWICK, D.G.: "Atypical small acinar proliferation for malignancy in prostate needle biopsies. Clinical significance in 33 cases". Am. J. Surg. Pathol.; 21: 1489. 1997.

35. GRACÍA GONZÁLEZ, R.; MAYAYO DEHESA, T.; LENNIE ZUCCARINO, A. y cols.: "Repetición de la biopsia prostática ecodirigida para la detección de cáncer. Estudio de una serie de 192 pacientes re-biopsiados". Actas Urol. Esp.; 24: 644. 2000.

36. ICZKOWSKI, K.A.; BOSTWICK, D.G.: "Criteria for biopsy diagnosis of minimal volumen prostatic adenocarcinoma. Analytic comparison with nondiagnostic but suspicious atypical small acinar proliferation". Arch. Pathol. Lab. Med.; 124: 98. 2000.

37. MAYAYO DEHESA, T.; RODRÍGUEZ-PATRÓN, R.; LENNIE ZUCCARINO, A. y cols.: "Análisis de los resultados en una serie de 1900 pacientes". Arch. Esp. Urol.; 52: 453. 1999. 